\title{
Clasificación de las fracturas toracolumbares: comparación entre las clasificaciones de AO y Vaccaro
}

\author{
Classification of thoracolumbar fractures: a comparison between the \\ classifications of $A O$ versus Vaccaro
} Classificação das fraturas toracolombares: comparação entre as classificações de $A O$ e Vaccaro

\author{
Pedro Luis Bazán' \\ Alvaro Enrique Borri² \\ Piscinis Ulises Torres ${ }^{3}$ \\ Juan Sebastián Cosentino ${ }^{4}$ \\ Martin Honorio Games ${ }^{5}$
}

\section{RESUMEN}

Objetivo: las clasificaciones han cambiado en el último medio siglo, la más usada desde la pasada década es la Clasificación AO. En 2004, Vaccaro et al. propusieron el Thoraco-Lumbar Injury Classification and Severity Score (TLICS). Métodos: análisis de la reproductividad inter $\mathrm{y}$ intra observador, utilizando el test Kappa, de las dos clasificaciones entre tres niveles distintos de traumatólogos en formación, en 30 casos. Resultados: la reproductividad intraobservador en la clasificación de Vaccaro fue: OI: 0,73 ; OII: 0,6 y OIII: 0,63 . Para la clasificación AO: 0,$7 ; 0,7$ y 0,6 , respectivamente. Entre las dos clasificaciones: OI: 0,59 ; OII: 0,7 y OIII: 0,62. La evaluación interobservador para la clasificación de Vaccaro es 0,66 y para la clasificación $\mathrm{AO}$ de 0,67 . Los puntos críticos: rotación y lesiones del complejo ligamentario posterior. Conclusiones: las dos clasificaciones muestran un buen grado de acuerdo (índice de Kappa). Con la de Vaccaro, se observó un acuerdo global del $69 \%$. Con respecto a la indicación

\section{ABSTRACT}

Objective: the classifications have changed in the last half century; the most used from the last decade is Classification AO. In 2004, Vaccaro et al. proposed the Thoraco-Lumbar Injury Classification and Severity Score (TLICS). Methods: one analysis of the inter and intra observant reproduction by using the Kappa test, of the two classifications between three different levels from orthopedic surgeons in formation in 30 cases. Results: the intraobservant reproduction in the classification of Vaccaro was: OI: 0.73; OII: 0.6, and OIII: 0.63 . For the AO classification: $0.7 ; 0.7$ and 0.6, respectively. Between the two classifications: OI: 0.59 ; OII: 0.7, and OIII: 0.62. The interobservant evaluation for the classification of Vaccaro is 0.66 and for AO classification was 0.67 . The tactically important points were rotation and injuries of the later ligamentary complex. Conclusions: the two classifications show a good degree in agreement (Kappa index). With the Vaccaro, it

\section{RESUMO}

Objetivo: as classificações têm mudado na último metade do século, sendo a mais usada desde a última década, a classificação AO. Em 2004, Vaccaro et al. propuseram a Thoraco-Lumbar Injury Classification (TLICS). Métodos: análise da reprodução inter e intraobservador, utilizando o teste Kappa das classificações entre três níveis distintos de traumatólogos em formação, em 30 casos. Resultados: a reprodução intraobservador na classificação de Vaccaro foi: OI: 0,73; OII: 0,6 e OIII: 0,63. Para a classificação AO, 0,77; 0,7 e 0,6, respectivamente. Entre as duas classificações: OI: 0,59; OII: 0,7 e OIII: 0,62. A avaliação interobservador para a classificação de Vaccaro foi de 0,66 e para a classificação AO de 0,67. Os pontos críticos foram rotação e lesões do complexo ligamentar posterior. Conclusões: as duas classificações mostram um bom grau de concordância (indice Kappa). Com a de Vaccaro, observou-se concordância global de 69\%. Com respeito à indicação do

\footnotetext{
Trabajo realizado en Hospital HIGA "San Martín", La Plata, Argentina
}

"Hospital HIGA "San Martín", La Plata, Argentina. 
de tratamiento ortopédico, el acuerdo fue el $37 \%$. En la indicación de tratamiento quirúrgico, fue del $29 \%$. Cabe remarcar que dicha clasificación dispone de un nivel impreciso donde se puede optar por cualquiera de los dos tratamientos (TLICS 4), esto se observó en el $3 \%$. No hubo concordancia en el $31 \%$. Las mismas indicaciones para la clasificación AO presentaron un acuerdo global del $67 \%$. Para la indicación ortopédica fue del $32 \%$. Se realizó una indicación quirúrgica en el $21 \%$. En las lesiones clasificadas como A3 (14\%), su indicación de tratamiento no es definida con unanimidad entre los observadores. has been observed a global agreement of $69 \%$. Regarding the indication of orthopedic treatment, the agreement was $37 \%$. In the surgical treatment indication, it was $29 \%$. It is possible to remark that this classification has a vague level where it can be decided on any of the two treatments (TLICS 4). This was observed in 3\%. There was no agreement in $31 \%$. The same indications for classification $A O$ presented a global agreement of $67 \%$. For the orthopedic indication, it was $32 \%$. A surgical indication was made in $21 \%$. In the injuries classified as A3 (14\%) about the treatment is not defined with unanimity between the observers. tratamento ortopédico, a concordância foi de 37\%. A indicação de tratamento cirúrgico foi de $29 \%$. Cabe salientar que essa classificação dispõe de um nível impreciso em que pode-se optar por qualquer dos dois tratamentos (TLICS 4), o qual foi observado em $3 \%$. Não houve concordância em 31\%. As mesmas indicações para a classificação AO apresentaram concordância global de $67 \%$. Indicação ortopédica foi de $32 \%$. Foi realizada uma cirurgia em $21 \%$. Nas lesões classificadas como A3 (14\%), a sua indicação de tratamento não foi definida com unanimidade entre os observadores.
DESCRIPTORES: Fracturas espinales/clasificación; Vértebras lumbares; Vértebras torácicas
KEYWORDS: Spinal fractures/

classification; Lumbar

vertebrae; Thoracic vertebrae
DESCRITORES: Fraturas da coluna vertebral/classificação; Vértebras lombares; Vértebras torácicas

\section{INTRODUCCIÓN}

La clasificación de las fracturas toracolumbares ha evolucionado durante los últimos 40 años. En 1949, Nicoll describió estas fracturas en relación a patrones de estabilidad según la indemnidad o rotura de los ligamentos que unen a vértebras contiguas $y$, sobre la base de ello, estableció directivas precisas de tratamiento ${ }^{1,2}$.

Holdsworth, en 1963, modificó y amplió la clasificación de Nicoll, y esta modificación constituye el pilar de todas las clasificaciones posteriores. Holdsworth las clasificó en cinco grupos según el mecanismo de lesión ${ }^{3}$, sugirió entonces dividir al raquis en dos columnas, una columna anterior compuesta por el cuerpo vertebral, el disco intervertebral y los ligamentos adyacentes; y una columna posterior, compuesta por los pedículos, el arco neural, las apófisis articulares con sus cápsulas y los ligamentos asociados ${ }^{4}$.

En 1983, Denis desarrolló el concepto de las tres columnas; luego de analizar una serie de más de 400 tomografías computarizadas (TC) de lesiones toracolumbares, sostuvo que había una columna anterior compuesta por la mitad anterior del cuerpo, el disco y el ligamento vertebral común anterior; una columna media formada por la mitad posterior del cuerpo, el disco y el ligamento vertebral común posterior; y una columna posterior igual que la descripta por Holdsworth ${ }^{2,4}$ (Figura 1).

Denis observó que una o más de las tres columnas fracasaban de forma previsible en la compresión axial, la distracción axial o la traslación por combinaciones de fuerzas en planos diferentes ${ }^{3,4}$. Esta premisa ha sido refutada por

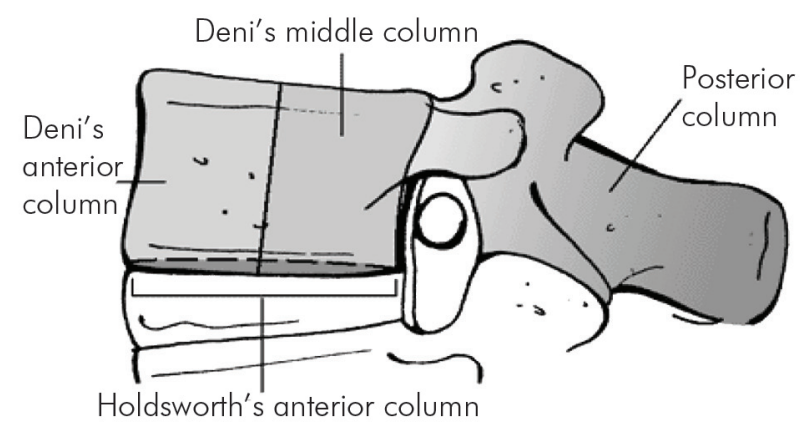

Figura 1

División en columnas según Denis y Holdsworth.

Fuente: Bucholz RW, Heckman JD, Court-Brown CM, Tornetta P, Koval KJ, editors. Rockwood \& Green's fractures in adult. 6th ed. New York: Lippincott Williams \& Wilkins; 2006. p. 1549.

varios trabajos que describen fracturas tipo estallido tratadas exitosamente de forma ortopédica. Todo lo descripto sumado a análisis recientes que le otorgan bajos niveles de confiabilidad y reproductibilidad hacen que la clasificación de Denis haya perdido vigencia ${ }^{4,5}$.

$\mathrm{Al}$ reconocer los problemas del sistema propuesto por Denis, en un estudio de las TC de 100 pacientes consecutivos con fracturas potencialmente inestables y fracturas - luxaciones, McAfee et al. determinaron el mecanismo de lesión del complejo osteoligamentoso y desarrollaron un nuevo sistema de clasificación basado en estos mecanismos. Este sistema fue propuesto previo a la introducción 
de la instrumentación con tornillos pediculares y barras, cuando los sistemas en gancho eran los más usados, estableciendo si las lesiones debían ser fijadas en distracción o compresión ${ }^{2,4}$.

Para muchos autores, la clasificación más completa, confiable y reproducible disponible en la actualidad es la propuesta por Magerl et al., en 1994, quienes revisaron las laminas de radiografías (Rx) y la TC de 1.445 lesiones toracolumbares consecutivas en cinco centros de trauma. Ellos describen tres tipos básicos según el mecanismo primario de producción: A) compresión, B) distracción y C) torsión o rotación. A su vez, éstos se dividieron en tres subgrupos, cada uno con tres subdivisiones ${ }^{4,6}$ (Figura 2).

Para algunos autores, al igual que lo que ocurre con la clasificación de Denis, la confiabilidad y reproductibilidad de este sistema es limitada ${ }^{4,5,7}$.

Luego de la introducción de los sistemas con tornillos pediculares y barras, se popularizó la estabilización por vía posterior de las fracturas toracolumbares por estallido; se realizaba la misma fijando tan solo un nivel por arriba y por debajo de la lesión ${ }^{4}$.

Numerosos trabajos publicados posteriormente demostraron altos índices de fracaso con este tipo de estabilización tan simplificada, lo que motivó a McCormack et al. a crearen una nueva clasificación orientada a predecir en qué casos esta configuración podría fallar, dado el alto grado de conminución de estas fracturas. Asignaron, entonces, diversos valores al grado de conminución vertebral, la aposición de los fragmentos y la cifosis, concluyendo que las lesiones con siete o más puntos serían mucho mejor tratadas si se realizaba también la reconstrucción por vía anterior ${ }^{4,7}$.

Estudios recientes demuestran que esta clasificación tiene un alto nivel de confiabilidad y reproductibilidad, comprobado también mediante estudios biomecánicos in vitro realizados sobre ganado bovino ${ }^{8,9}$.

A partir de la controversia existente en relación a la clasificación y el manejo de las fracturas toracolumbares, Vaccaro et al. desarrollaron en el año 2004 un nuevo sistema de clasificación y valoración de la severidad de estas lesiones: "The Thoraco-lumbar Injury and Severity Score" (TLISS), basado en tres variables mayores que son: 1) el mecanismo de producción según $\mathrm{Rx}, 2$ ) indemnidad del complejo ligamentario posterior y 3) estado neurológico del paciente; proponiendo un algoritmo terapéutico en cada caso particular ${ }^{10}$.

El primer paso supone determinar el mecanismo de producción según el patrón encontrado en las imágenes obtenidas. Las lesiones se describen usualmente en tres categorías similares a las de la clasificación AO: lesiones por compresión, lesiones por traslación/rotación y lesiones por distracción. Se asignan de 1 a 4 puntos, reflejando la severidad del compromiso ${ }^{10,11}$ (Tabla 1 ).

\section{TABLA 1 - Puntuación de la Clasificación}

\section{Vaccaro}

\begin{tabular}{lcc}
\hline Morfología & Calificación & Puntos \\
\hline Compresión & Estallido & 1 \\
& & 3 \\
Traslación/rotación & & 4 \\
Distracción & \\
Estado neurológico & & 0 \\
Intacto & & 2 \\
Lesión radicular & Incompleto & 3 \\
Médula o cono medular & Completo & 2 \\
& & 3 \\
Cauda equina & & \\
Complejo ligamentario posterior & & 0 \\
Intacto & & 2 \\
Sospechoso/dudoso & & 3 \\
Lesionado & & \\
\hline
\end{tabular}

Una vez que se han asignado valores a las tres variables mayores, se obtiene un total de puntos que orienta hacia el manejo ortopédico o quirúrgico de estas lesiones. Los pacientes con tres puntos o menos son considerados candidatos al tratamiento ortopédico; los pacientes con cinco
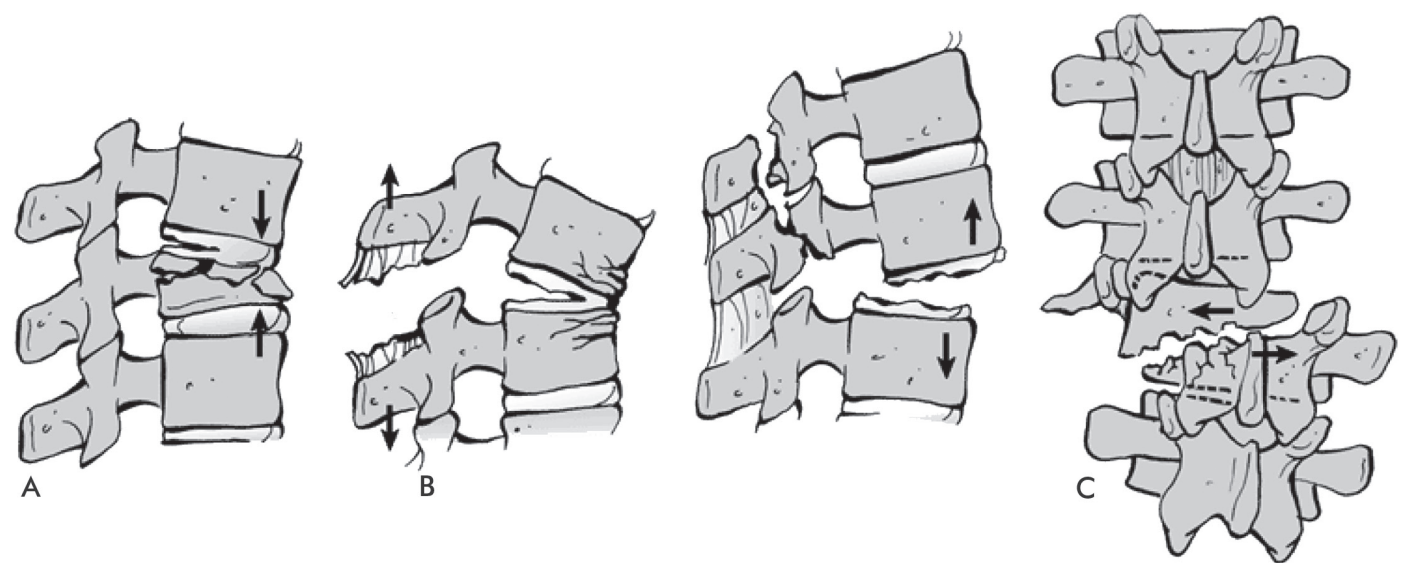

Figura 2

Clasificación AO.

Fuente: Magerl F, Aebi M, Gertzbein SD, Harms J, Nazarian S. A comprehensive classification of thoracic and lumbar injuries. Eur Spine J. 1994;3(4): 184-201. 
o más puntos son considerados candidatos al tratamiento quirúrgico y en los pacientes con cuatro puntos pueden considerarse ambas opciones terapéuticas ${ }^{10,12}$.

En cuanto a su validación y confiabilidad, se han realizado varios estudios que han culminado con la creación de una variable del TLISS llamada TLICS, "Thoraco-lumbar Injury Classification and Severity Score", que evalúa la morfología de la lesión con más exactitud y tiene niveles similares de confiabilidad y reproductibilidad. Esta clasificación también orienta hacia el abordaje quirúrgico, siendo los dos principales factores a considerar la indemnidad del complejo ligamentario posterior y el estado neurológico del paciente ${ }^{13}$.

Los principios generales del tratamiento quirúrgico serían: una lesión neurológica incompleta requiere realizar una vía anterior si presenta compresión a partir de elementos anteriores; la lesión del complejo ligamentario posterior generalmente se aborda por vía posterior; y una combinación de los dos anteriores requiere también un doble abordaje $\mathrm{e}^{13,14}$.

En comparación con los sistemas y clasificaciones previos, tanto el TLISS como el TLICS han demostrado ser fáciles de aplicar y son la mejor alternativa disponible para el manejo de las lesiones toracolumbares, con niveles de reproductibilidad mucho mayores que las clasificaciones de Denis y de la $\mathrm{AO}^{14}$.

Aunque los últimos trabajos disponibles se inclinan ligeramente a favor de la confiabilidad del TLISS, aclaran que ambos conceptos deberían tenerse en consideración, tanto el mecanismo de producción de la lesión como la morfología del trazo de fractura, ya que ambos están íntimamente relacionados y son críticos para el mantenimiento de la estabilidad de la columna ${ }^{15}$. Los dos son sistemas con excelente validación y configuran una herramienta útil para el manejo de las lesiones toracolumbares ${ }^{14,15}$.

La mayoría de las clasificaciones fueron implementadas para describir los patrones lesiónales. Algunas permiten también realizar un pronóstico del paciente. Sin embargo, cualquier sistema debería ser fácil de recordar y aplicable en la práctica médica cotidiana, permitiendo determinar la severidad de la lesión y guiar la decisión del tratamiento a seguir.

El objetivo de este trabajo es comparar la confiabilidad y reproductibilidad de la clasificación AO con la clasificación propuesta por Vaccaro entre residentes de Ortopedia y Traumatología y especialistas de traumatología dedicados a la patología espinal.

\section{MÉTODOS}

Se realiza el análisis retrospectivo de las imágenes de radiografías, tomografía computada y/o resonancia magnética de 30 pacientes ya tratados, en forma ortopédica o quirúrgica, por presentar una fractura o fractura luxación de columna toracolumbar.

Cada caso fue clasificado en primer lugar de acuerdo a la clasificación de Magerls et al. (clasificación AO) y la propuesta por Vaccaro et al. (TLICS), en primer lugar por un especialista en Ortopedia y Traumatología dedicado a la Patología Espinal (AI) y por un practicante en patología espinal post-residencia.

Posteriormente, en forma ciega, en total de diez casos y en tres oportunidades por vez, se realizó la clasificación por parte de un residente con rotación completa en la Unidad de Patología Espinal (OI), otro con la rotación en curso (OII) y un tercero que no había realizado su rotación respectiva (OIII). A todos los residentes se los condicionó en el manejo y utilización de ambas clasificaciones, con la utilización de algoritmo de correcto uso (Figuras 3 y 4).

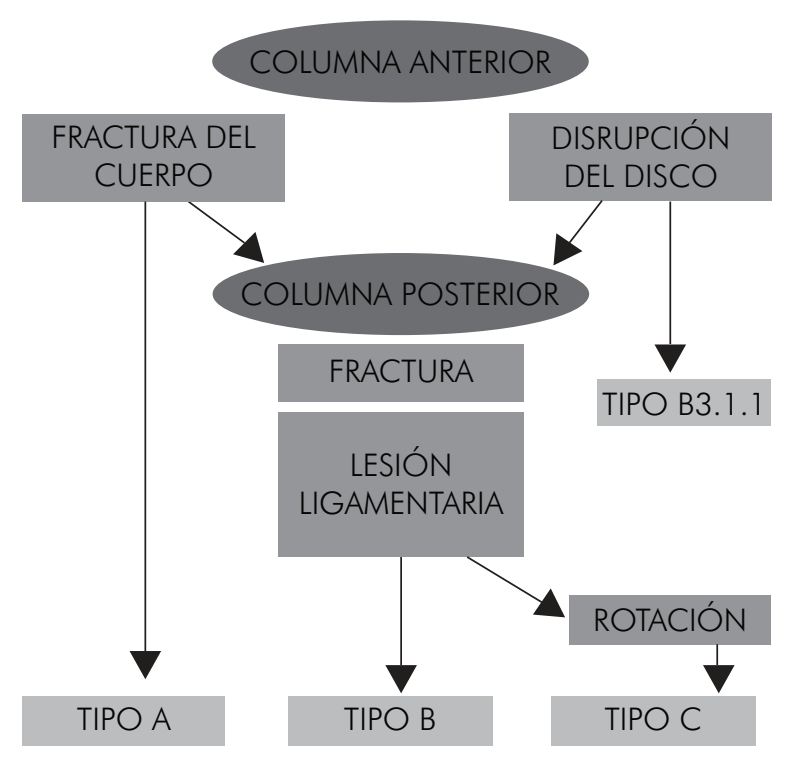

Figura 3

Algoritmo de utilización de la Clasificación AO.

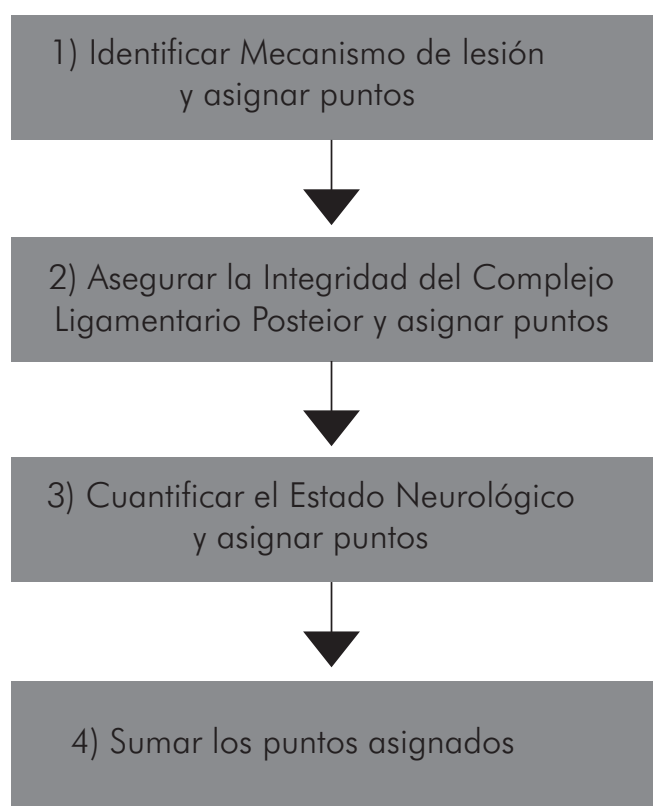

Figura 4

Algoritmo de utilización de la Clasificación Vaccaro. 
La primera clasificación utilizada fue la de Vaccaro y, una vez analizados los 30 pacientes, los participantes entregaban una planilla preparada para volcar los datos. Luego de analizar en tres oportunidades y habiendo entregado sus respectivas planillas, se realizó la misma metodología con la clasificación AO.

Para el análisis de los resultados y precisar su confiabilidad, se utilizó el índice de Kappa (Tabla 2) comparando reproductividad intra e interobservador, comparando el grupo de residentes y luego de ellos con los otros dos autores en conjunto.

TABLA 2 - Grado de acuerdo según índice de Kappa

\begin{tabular}{lc}
\hline Kappa & Grado de acuerdo \\
\hline$<0$ & Sin acuerdo \\
$0-0,2$ & Insignificante \\
$0,2-0,4$ & Bajo \\
$0,4-0,6$ & Moderado \\
$0,6-0,8$ & Bueno \\
$0,8-1$ & Muy bueno \\
\hline
\end{tabular}

\section{RESULTADOS}

La reproductividad intraobservador observada en la clasificación de Vaccaro fue: OI: 0,73; OII: $0,6 \mathrm{y}$ OIII: 0,63. Para la clasificación AO fue: 0,$7 ; 0,7$ y 0,6 , respectivamente.

Cuando se comparan los datos obtenidos entre ambas clasificación en el mismo observador, se obtuvieron los siguientes resultados; OI: 0,59; OII: 0,7 y OIII: 0,62. (Tabla 3).

TABLA 3 - Reproductividad intraobservador utilizando el índice de Kappa

\begin{tabular}{lccc}
\hline Observador & Vaccaro & AO & Vaccaro/AO \\
\hline OI & 0,73 & 0,7 & 0,59 \\
Oll & 0,60 & 0,7 & 0,70 \\
Olll & 0,63 & 0,6 & 0,62 \\
\hline
\end{tabular}

El índice de reproductividad hallado evaluando la diferencia interobservador para la clasificación de Vaccaro fue de 0,66 , y para la clasificación $\mathrm{AO}$ de 0,67 . Al relacionarlo con los autores desciende a 0,50 y 0,49 , respectivamente, siendo en los puntos críticos la rotación y lesiones del complejo ligamentario posterior.

\section{DISCUSIÓN}

En ambas clasificaciones analizadas existen puntos de no acuerdo en el tratamiento a indicar, así como se observa en del punto 4 de TLICS donde se puede optar entre la terapéutica ortopédica o quirúrgica ${ }^{5,6,8,10,13}$.

La misma problemática se observa en las clasificadas como A3, algunas A2 o pocas B2. En todos los casos se toman como marco de referencia la presencia de déficit neurológico, compromiso del canal mayor a $50 \%$, cifosis más de $30^{\circ}$ para las $\mathrm{A} 3^{6,12}$.

Observando que ambas clasificaciones presentan una buena reproductibilidad y confiabilidad creemos que la clasificación de Vaccaro (TLICS) permite una interpretación más fácil y completa a médicos no habituados en lesiones raquimedulares.

\section{CONCLUSIONES}

Las dos clasificaciones evaluadas muestran un buen grado de acuerdo utilizando el índice de Kappa, para la evaluación inter e intraobservador.

En la clasificación de Vaccaro, se observó un acuerdo global del $69 \%$. Con respecto a la indicación de tratamiento ortopédico (TLICS $<4$ ), se presentó un acuerdo del $37 \%$ entre los distintos autores. En la indicación de tratamiento quirúrgico (TLICS $>4$ ), el acuerdo fue del $29 \%$. Cabe remarcar que dicha clasificación dispone de un nivel impreciso donde se puede optar por cualquiera de los dos tratamientos (TLICS 4), esto se observó en el 3\% de la evaluación. No hubo concordancia en la clasificación en el $31 \%$ de las determinaciones.

Las mismas indicaciones para la clasificación AO presentaron un acuerdo global del 67\%. Para la indicación ortopédica (A1 y A2), fue del 32\%. Se realizó una indicación quirúrgica $(\mathrm{B}$ y $\mathrm{C}$ ) en el 21\%. En las lesiones clasificadas como A3 (14\%), su indicación de tratamiento no es definida con unanimidad entre los observadores.

\section{REFERÊNCIAS}

1. Cosentino R. Fracturas y luxaciones de la columna vertebral. En: Cosentino R. Raquis, Semiología. 2 ed. Buenos Aires: El Ateneo; 1986. p. 215-23.
2. McLain RF, Benson DR. Thoracolumbar Fractures: Evaluation, Classification and Initial Management. In: Chapman's Orthopaedic Surgery. 3rd ed. Lippincott Williams \& Wilkins; 2001. p. 3714-725.
3. Leventhal MR. Fracturas, Luxaciones y Fracturas-luxaciones de la Columna. En: Crenshaw $\mathrm{AH}$, Daugherty K, Curro $\mathrm{CH}$, eds. Campbell. Cirugía Ortopédica. 10 ed., vol. 2, Buenos Aires: Editorial Médica Panamericana; 2004. p. 1642-4. 
4. Eastlack RK, Bono CM.

Fractures and Dislocations of the Thoracolumbar Spine. En: Bucholz RW, Heckman JD, Court-Brown C, eds. Rockwood \& Green's Fractures in Adults. 6 ed. Philadelphia: Lippincott Williams \& Wilkins; 2006. p. 1544-80

5. Vaccaro AR, Lim MR, Hurlbert RJ, Lehman RA Jr, Harrop J, Fisher DC, et al. Surgical decision making for unstable thoracolumbar spine injuries: results of a consensus panel review by the Spine Trauma Study Group. J Spinal Disord Tech. 2006;19(1):1-10.

6. Magerl F, Aebi M, Gertzbein SD, Harms J, Nazarian S. A comprehensive classification of thoracic and lumbar injuries. Eur Spine J. 1994;3(4):184-201.

7. Aligizakis AC, Katonis PG, Sapkas G, Papagelopoulos PJ, Galanakis I, Hadjipavlou A. Gertzbein and load sharing classifications for unstable thoracolumbar fractures. Clin Orthop Relat Res. 2003;(411):77-85.

8. Vaccaro AR, Zeiller SC, Hulbert RJ, Anderson PA, Harris M, Hedlund $\mathrm{R}$, et al. The thoracolumbar injury severity score: a proposed treatment algorithm. J Spinal Disord Tech. 2005;18(3):209-15.
9. Bono CM, Vaccaro AR, Hurlbert RJ, Arnold P, Oner FC, Harrop $\mathrm{J}$, et al. Validating a newly proposed classification system for thoracolumbar spine trauma: looking to the future of the thoracolumbar injury classification and severity score. J Orthop Trauma. 2006;20(8):567-72.

10. Vaccaro AR, Lehman RA Jr, Hurlbert RJ, Anderson PA, Harris M, Hedlund R, et al.: A new classification of thoracolumbar injuries the importance of injury morphology, the integrity of the posterior ligamentous complex, and neurologic status. Spine (Phila Pa 1976). 2005;30(20):2325-33.

11. Dai LY, Jin WJ. Interobserver and intraobserver reliability in the load sharing classification of the assessment of thoracolumbar burst fractures. Spine 2005; 30(3):354-8.

12. Wood KB, Khanna G, Vaccaro AR, Arnold PM, Harris MB, Mehbod AA. Assessment of two thoracolumbar fracture classification systems as used by multiple surgeons. J Bone Joint Surg Am. 2005;87(7):1423-9.
13. Vaccaro AR, Baron EM, Sanfilippo J, Jacoby S, Steuve J, Grossman E, et al. Reliability of a novel classification system for thoracolumbar injuries: the Thoracolumbar Injury Severity Score. Spine (Phila Pa 1976). 2006;31(11 Suppl):62-9.

14. Wang XY, Dai LY, Xu HZ, Chi YL. The load-sharing classification of thoracolumbar fractures: an in vitro biomechanical validation. Spine (Phila Pa 1976). 2007;32(11): 1214-9.

15. Whang PG, Vaccaro AR, Poelstra KA, Patel AA, Anderson DG, Albert $\mathrm{TJ}$, et al. The influence of fracture mechanism and morphology on the reliability and validity of two novel thoracolumbar injury classification systems. Spine (Phila Pa 1976). 2007;32(7):791-5.

\section{Correspondência}

Pedro Luis Bazán

Direccion 1y 70 La Plata, Provincia de Buenos Aires - Argentina

E-mail: pbazan@hilp.org.ar 